OPEN ACCESS

Edited by:

Jorge Amil Dias,

Centro Hospitalar de São João,

Portugal

Reviewed by:

Iva Hojsak,

Children's Hospital Zagreb, Croatia

Paula Maria Guerra

São João University Hospital

Center, Portugal

${ }^{*}$ Correspondence:

Hsun-Chin Chao

hcchao1021@gmail.com

Specialty section: This article was submitted to

Pediatric Gastroenterology, Hepatology and Nutrition,

a section of the journal

Frontiers in Pediatrics

Received: 26 December 2020 Accepted: 08 February 2021 Published: 18 March 2021

Citation:

Chen M-C, Chao H-C, Yeh P-J,

Lai M-W and Chen C-C (2021)

Therapeutic Efficacy of Nasoenteric

Tube Feeding in Children Needing

Enteral Nutrition.

Front. Pediatr. 9:646395.

doi: 10.3389/fped.2021.646395

\section{Therapeutic Efficacy of Nasoenteric Tube Feeding in Children Needing Enteral Nutrition}

\author{
Mi-Chi Chen ${ }^{1}$, Hsun-Chin Chao ${ }^{1,2 *}$, Pai-Jui Yeh ${ }^{1}$, Ming-Wei Lai ${ }^{1,2}$ and Chien-Chang Chen ${ }^{1,2}$ \\ ${ }^{1}$ Division of Pediatric Gastroenterology, Department of Pediatrics, Chang Gung Children's Medical Center, Chang Gung \\ Memorial Hospital, Taoyuan City, Taiwan, ${ }^{2}$ Chang Gung University College of Medicine, Taoyuan City, Taiwan
}

Background: There is limited information on therapeutic benefits and tube-related complications of pediatric nasoenteric (NE) tube feeding. We viewed, from different clinical aspects, NE tube feeding in children who are under intolerable conditions.

Methods: A 10-years retrospective study enrolled 77 pediatric patients who underwent an endoscopic-guided placement of the NE tube for enteral nutrition. The evaluated data, including growth parameters, feeding volume, parenteral nutrition (PN) dependence, and nutritional markers [serum hemoglobin $(\mathrm{Hb})$ and albumin] before and after NE tube feeding were compared. Tube-related complications and major adverse events were also recorded.

Results: A total of 77 patients (including 50 males) underwent 176 endoscopic-guided placements of the NE tube with an average duration of $133.7(6.0-1,847.3)$ days. The gastroesophageal reflux disease-related symptoms (vomiting, desaturations, and aspiration pneumonia) improved in $71.4 \%$ of patients. Feeding volume increased significantly after intervention, especially in patients with delayed gastric emptying, from $144.8 \pm 28.5$ to $1,103.1 \pm 524.7 \mathrm{ml} /$ days $(p<0.001)$. Weaning from PN was successfully achieved in $84.3 \%$ of patients with an average of $9.33 \pm 7.30$ days. About 16 patients (20.8\%) were subsequently highly compatible with oral feeding after NE tube placement for an average of $24.7 \pm 14.1$ days. Patients either without neurologic dysfunction or with no ventilator-dependent status had a higher chance of shifting to oral feeding. Weight-for-age $z$-scores increased by $0.15 \pm 1.33$ after NE tube intervention. One NE tube-related adverse event, which caused bowel perforation at 6 days post-insertion, was recorded. No direct tube-related mortality was observed.

Conclusions: Endoscopic-guided NE tube placement is a relatively safe, non-invasive procedure for pediatric patients who require enteral nutrition. Feeding via NE tube showed beneficial effects such as improvement in symptoms, PN weaning, and maintenance of body growth without major tube-related complications.

Keywords: children, enteral nutrition, naso-enteric tubes, nutritional status, gastrointestinal reflux 


\section{INTRODUCTION}

Nutritional support is indicated for patients with inadequate nutrition intake or manifestation of wasting and stunting (1). Enteral feeding is more favorable than parenteral feeding in patients with a functioning digestive tract because the former can maintain gut integrity and prevent bacterial translocation (2). Different tube feeding strategies are available for short- or longterm use. In general, nasogastric (NG) tube is the most common and easiest route for pediatric patients to provide nutrition support (3).

Enteral tube feeding involves the artificial delivery of nutrition directly to the gastrointestinal tract without the need for swallowing. In temporary or short-term situations, this method is most commonly performed via an NG tube into the stomach but can be achieved via post-pyloric access with a nasoenteric (NE) tube (nasoduodenal or nasojejunal tube) into the proximal small bowel (4). Post-pyloric access is indicated in specific situations, such as severe gastrointestinal reflux disease (GERD) with a risk of aspiration, gastric emptying dysfunction, gastric outlet obstruction, acute pancreatitis, and previous gastric surgery precluding gastric feeding or in early postoperative feeding after major abdominal surgery $(1,5,6)$. The common candidates for NE tube feeding include neurological disability, critically ill children, and young infants (6).

Several studies established the efficacy of small bowel feeding for nutritional support, and growing evidence showed benefits for infants and children who fail to thrive under intragastric feeding $(6,7)$. The present study aims to investigate the clinical outcomes of pediatric patients who received enteral nutrition via endoscopic-guided NE tube feeding in a tertiary hospital.

\section{METHODS}

\section{Study Design}

Children who were aged $<18$ years and who underwent endoscopic-guided NE tube placement for enteral nutrition between January 2011 and June 2020 were enrolled in the study. Four indications used for NE tube placement are described as follows: (i) severe GERD with recurrent emesis despite prokinetic and antacid treatment, recurrent aspiration pneumonia, or frequent desaturation or bradycardia attack during or after feeding; (ii) delayed gastric emptying with gastric residuals of over $50 \%$ of the administered volume in the previous $4 \mathrm{~h}$ (8); (iii) post-surgery nutritional support with a functioning gut but with complete intolerance to oral or NG tube feeding within the first postoperative week; and (iv) partial obstruction of the upper gastrointestinal tract (UGI) demonstrated by using barium study or direct endoscopic visualization. Except for contraindications to NG feeding, most of the enrolled patients tried NG feeding but experienced intolerance or were unable to meet the nutritional requirements to achieve adequate body growth. We suggest gastrostomy (GT) (with or without Nissen fundoplication) or jejunostomy for patients who require long-term enteral nutrition for more than 4-6 weeks (9-11) and for some of our patients who had prolonged use of the NE tube for feeding due to unsuitability to or family's refusal to GT or jejunostomy for long-term feeding.
Medical records, including demographic information, underlying diseases, nutritional status (body weight, body height/length, and BMI), laboratory data [Hb, mean corpuscular volume (MCV), and albumin], and average daily feeding volume (assessed by a 3-days dietary record), were collected. The laboratory data and feeding volume at the initiation and at the end of NE tube feeding were compared. The data on the evaluated serum albumin were obtained at $2-3$ weeks before and 2-3 weeks after the end of NE tube feeding (12).

\section{The Procedure for Nasoenteric Tube Placement}

In our institution, we offer endoscopic-guided NE tube placement for patients who are unable to tolerate NG tube feeding for variable periods. Unweighted polyurethane NE tubes (CORFLO Enteral Feeding Tubes) with sizes of 6-12 French based on age and body weight were provided to the patients. The patients were sedated using intravenous midazolam $(0.1-$ $0.2 \mathrm{mg} / \mathrm{kg} / \mathrm{dose}$ ), and their vital signs during the procedure were monitored. The NE tube was placed beyond the second portion of the duodenum through the nose under endoscopic guidance $(5,13)$. The injection and withdrawal of dis-water were tested for function assurance. X-ray confirmation of a proper tube location before the initiation of feeding is mandatory at our institution (10). Ideally, the tip position is located beyond the ligament of Treitz and proximal jejunum. The nutritionist was advised with a feeding formula and feeding schedule individually. The scheduled time for exchanging NE tubes was every 3 months or the time until tube dysfunction.

\section{Outcome Measures}

The measured outcomes of NE tube feeding included nutritional promotion, successful replacement of parenteral nutrition (PN), improvement of symptoms, subsequent feeding strategy (oral, NG, and GT or jejunostomy) after discontinuation of NE tube feeding, and complications, such asdislodgement, occlusion, breaks, migration to incorrect position, refeeding syndrome, intussusception, bowel perforation, serious bacterial enterocolitis, and mortality). Nutritional promotion measurement included nutritional markers ( $\mathrm{Hb}$ and serum albumin level) and nutritional status. The nutritional status was measured by using weight-for-age $z$-score and height-for-age $z$-score, calculated using WHO Anthro v.3.2.2 software (World Health Organization, Geneva, Switzerland) in patients who were younger than 5 years old and using WHO AnthroPlus v.1.0.4 software (WHO, Geneva, Switzerland) in patients aging 5-19 years old. The weaning of PN was defined as full enteral feeding without any additional intravenous fluids and PN support. The improvement of symptoms would be defined as an occurrence of vomiting $<3$ times per week without interfering with the advancement of feeding volume and vital sign changes (desaturations and bradycardia) at $<1$ time per week. A partial improvement is defined as a partial relief but not reaching the abovementioned goals. Tube-related complications would be reviewed from medical records. 


\section{Statistical Analysis}

The continuous variables are expressed as mean $\pm \mathrm{SD}$, median, and interquartile ranges if they followed a non-normal distribution. Comparison results were analyzed with the Student's $t$-test (normally distributed continuous variables), the MannWhitney U test (non-normal distributed variables), and the Chisquared test (categorical variables). Status changes were analyzed with a paired $t$-test. Statistical analysis was performed by using Statistical Product and Service Solutions software version 24.0 (SPSS Inc., Chicago, IL, USA). A $p<0.05$ was considered statistically significant.

\section{Ethical Approval}

The study was approved by the Ethics Committee of the Chang Gung Memorial Hospital (Ref. 202001377B0).

\section{RESULTS}

\section{Patient Demographics}

Between January 2011 and June 2020, 83 cases received endoscopic-guided NE tube placement at the Chang Gung Memorial Hospital. About five of the 83 eligible patients were excluded due to short-term NE tube placement, and one patient was excluded due to non-endoscopic guidance. Table 1 summarizes the characteristics of study patients. The mean age was $4.3 \pm 5.41$ years, and the age of 34 patients $(44.2 \%)$ was $<1$ year. Exactly 49 patients $(63.6 \%)$ had neurologic dysfunction, including cerebral palsy, encephalitis, brain tumor, intracranial hemorrhage, and neurodevelopmental delay. About 25 patients (32.5\%) were born prematurely, and the average gestational age was 32.2 weeks (range: $26-36$ weeks).

\section{Experiences of Endoscopic-Guided NE Tube Placement}

In the study period, we performed 176 uneventful endoscopicassisted NE tube placements. The success rate was $98.9 \%$. The average duration of the placement of the NE tube was 133.7 days (range: 6-1,847). The most common indication was severe GERD $(N=42,54.5 \%)$, followed by delayed gastric emptying $(N=23$, $29.9 \%)$, partial UGI obstruction $(N=7,9.1 \%)$, and postoperative nutritional support $(N=5,6.5 \%)$. The GERD-related symptoms were vomiting refractory to medications $(N=31,73.8 \%)$, desaturations/bradycardia during/after feeding $(N=15,35.7 \%)$, aspiration pneumonia episode $(N=11,26.2 \%)$, and choking $(N=10,23.8 \%)$. The etiologies of patients with partial UGI obstruction $(N=7)$ included pylorus spasm $(N=2)$, antral web $(N=1)$, GT leakage $(N=1)$, trauma-related duodenal obstruction $(N=2)$, and superior mesenteric artery syndrome $(N=1)$. Four patients who required post-operation nutritional support $(N=5)$ were operated due to burn injuries, and one patient was operated due to necrotizing fasciitis.

\section{Therapeutic Efficacy of NE Tube in Place}

\section{(I) Symptom Improvement}

In the severe GERD group $(N=42), 30$ patients $(71.4 \%)$ showed an obvious improvement and seven patients $(16.7 \%)$ showed a partial improvement of vomiting and desaturations.
TABLE 1 | Patient demographics in patients with nasoenteric (NE) tube feeding.

\begin{tabular}{|c|c|}
\hline Parameters & Values \\
\hline Case number & 77 \\
\hline \multicolumn{2}{|l|}{ Age (years) } \\
\hline Mean \pm SD & $4.30 \pm 5.41$ \\
\hline Median, IQR & $1.24,6.34$ \\
\hline Male (\%) & $50(64.9 \%)$ \\
\hline \multicolumn{2}{|l|}{ Body mass parameters } \\
\hline \multicolumn{2}{|l|}{ Weight (kg) } \\
\hline Mean \pm SD & $13.59 \pm 36.71$ \\
\hline Median, IQR & $8.4,13.53$ \\
\hline Weight-for-age $z$-score, mean \pm SD & $-2.75 \pm 2.71$ \\
\hline \multicolumn{2}{|l|}{ Length/ height $(\mathrm{cm})$} \\
\hline Mean \pm SD & $86.47 \pm 36.71$ \\
\hline Median, IQR & $75.5,55$ \\
\hline Height-for-age $z$-score, mean $\pm S D$ & $-2.43 \pm 2.70$ \\
\hline \multicolumn{2}{|l|}{ Medical conditions/comorbidity } \\
\hline Ventilator use $\mathrm{e}^{\#}$ & $40(51.9 \%)$ \\
\hline Cardiovascular dysfunction* & $9(11.7 \%)$ \\
\hline Neurologic dysfunction & $49(63.6 \%)$ \\
\hline Preterm & $25(32.5 \%)$ \\
\hline Congenital abnormalities & $14(18.2 \%)$ \\
\hline Gl tract structural anomaly & $10(13 \%)$ \\
\hline Burn & $4(5.2 \%)$ \\
\hline Leukemia/Lymphoma & $3(3.9 \%)$ \\
\hline Inborn errors of metabolism & $2(2.6 \%)$ \\
\hline
\end{tabular}

$S D$, standard deviation; IQR, interquartile range.

\# Ventilator use: under mechanical ventilation, including non-invasive ventilator.

${ }^{*}$ Cardiovascular dysfunction: under inotropes agent support, including epinephrine, dopamine, Milrinone.

NE tube feeding also helped to reduce the risk of aspiration pneumonia. Two patients with frequent aspiration pneumonia showed decreased episodes after NE tube feeding (the reduction of episodes from three times in 1 year to eight times in 5 years and from five times in 5 years to one time in 3 years), whereas the other patients who had a previous history of aspiration pneumonia experienced no recurrence during the NE tube feeding.

Overall, the daily feeding volume significantly increased from $304.3 \pm 349.0$ to $861.0 \pm 497.8 \mathrm{ml} /$ days, $p<0.001$ (Figure 1 ). In the delayed gastric emptying group, patients exhibited a significant increase in the daily volume from $144.8 \pm 28.5$ to $1,103.1 \pm 524.7 \mathrm{ml} /$ day, $p<0.001$.

\section{(II) Weaning From Parenteral Nutrition}

About 32 patients (41.6\%) required PN support before NE tube feeding. About 27 (84.3\%) of 32 patients were weaned from PN successfully within 1 month with an average duration of $9.33 \pm$ 7.30 days. Among PN-dependent patients $(N=5)$, two of them failed to achieve adequate feeding volume with NE tube feeding after 11 and 17 days, and the tubes were removed. One patient had NE tube-related bowel perforation, and one patient with total intestinal hypoganglionosis-related short bowel syndrome 


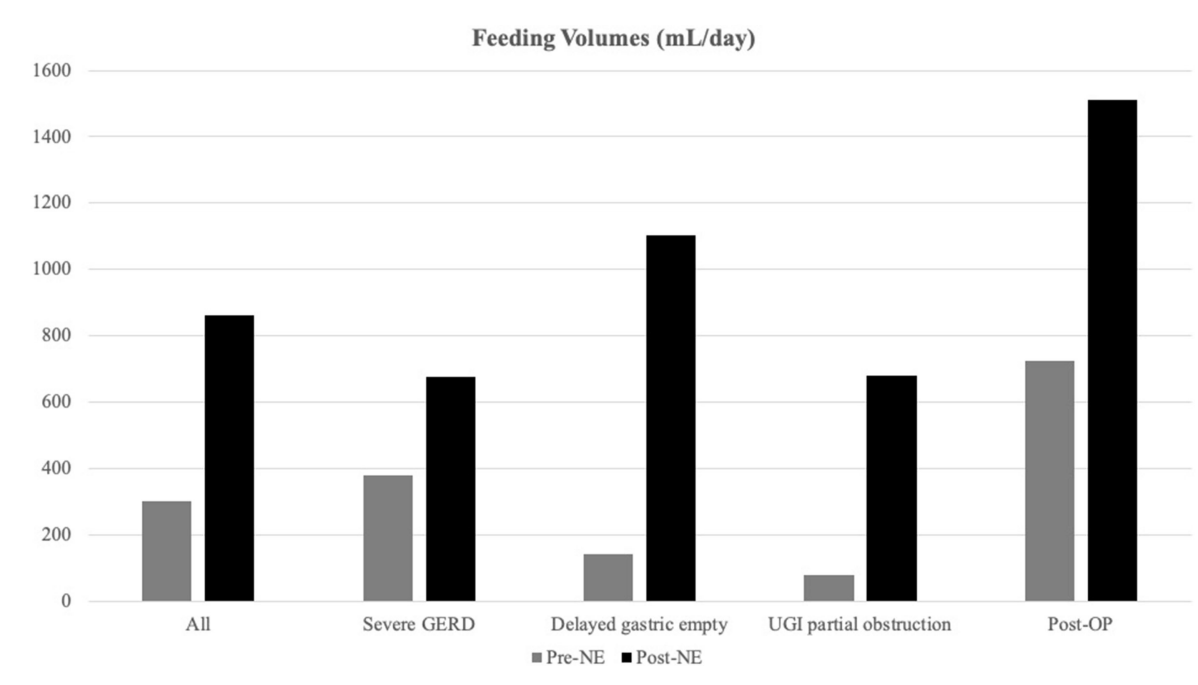

FIGURE 1 | Feeding volume alternations after nasoenteric (NE) tube feeding. Feeding volumes were increased significantly after NE tube placement, especially in the delayed gastric emptying group (from $144.8 \pm 136.5$ to $1,103.1 \pm 524.7 \mathrm{ml} /$ days) than the non-delayed gastric emptying group from $373.5 \pm 389.4$ to $755.9 \pm 451.3$ $\mathrm{ml} /$ days, $p<0.0001)$.

was dependent on PN. The fifth patient achieved full feeding but required a high protein intake due to severe burn injury.

\section{(III) Nutritional Laboratory Markers}

Serum $\mathrm{Hb}$ and albumin data of pre-NE and post-NE tubes in place were available for 67 and 35 patients, respectively. Mean pre- and post-NE tube $\mathrm{Hb}$ levels were $11.0 \pm 1.7 \mathrm{~g} / \mathrm{dl}$ and 11.3 $\pm 1.9 \mathrm{~g} / \mathrm{dl}$, respectively. The serum albumin level revealed a significant elevation from $3.22 \pm 0.84$ to $3.82 \pm 0.67 \mathrm{~g} / \mathrm{dl}$ after the intervention.

\section{(IV) Growth Assessment}

The average weight-for-age $z$-score for patients was $-2.75 \pm$ 2.71 and the average height-for-age $z$-score for patients was $-2.43 \pm 2.70$. After NE tube placement, the patients exhibited an increment in weight-for-age $z$-score of value $0.15 \pm 1.33$ and in height-for-age $z$-score of value $-0.27 \pm 1.26$ without statistical significance.

Patients with severe underweight (weight-for-age $z$-score $<-3$ ) and severe stunting (height-for-age $z$-score $<-3$ ) accounted for 45.5 and $26.0 \%$, respectively. We stratified patients with baseline weight-for-age $z$-score for three groups (Figure 2): non-malnourished ( $z$-score more than $-2, N=30$ ), moderate underweight ( $z$-score between -3 and $-2, N=8$ ), and severe underweight $(z$-score $<-3, N=35)$, and weight-for-age $z$-score increments for the three groups were $0.03 \pm 1.33$ ( $p=0.889), 0.25$ $\pm 0.62(p=0.292)$, and $0.23 \pm 1.47(p=0.361)$, respectively.

We further analyzed the growth of patients who had prolonged use of NE tube feeding for over 3 months (Figure 3). The average weight-for-age $z$-score and height-for-age $z$-score increased from $-4.02 \pm 2.87$ to $-3.42 \pm 3.84$ and from -3.05 \pm 2.56 to $-2.97 \pm 2.13$, respectively, in 1 year.

\section{Subsequent Feeding Strategy}

Subsequent feeding strategiesy are summarized in Table 2. Overall, 16 patients (20.8\%) experienced the successful removal of the NE tubes and tolerated oral feeding well with an average of $24.7 \pm 14.1$ days (range: 6-68 days). Patients without neurologic dysfunction had a higher rate of changing to oral feeding than those with neurologic dysfunction (41.7 vs. 6.1\%, $p<0.001$ ). Patients who were initially under ventilator support had a lower chance of shift to oral feeding than those without the support (2.6 vs. $35.3 \%, p<0.001)$.

About 30 patients (40.0\%) were weaned from the NE tube to an NG tube. Among these patients, 13 (43.3\%) received the NE tube because of delayed gastric emptying, indicating the mostly transient gastric emptying dysfunction. Eight patients (10.4\%) subsequently received surgical GT, and seven patients received Nissen fundoplication during the same surgery. The GT indications were recurrent emesis despite $\mathrm{NE}$ tube feeding $(N=7)$ and aspiration pneumonia $(N=1)$. Six patients continued receiving $\mathrm{NE}$ tube feeding and nine patients were lost to follow-up at the end of the study. Seven mortality cases $(9.1 \%)$ were observed in the present study because of sepsis $(N=6)$ and uncal herniation $(N=1)$.

\section{Outcomes of Short- and Long-Term Placements of Nasoenteric Tubes}

Short- $(<8$ weeks) and long-term placements of NE tubes are compared and summarized in Table 3. In the longterm group, most of the patients $(79.3 \%)$ had neurologic dysfunction, and the baseline weight-for-age $z$-score and height-for-age $z$-score were lower than that of the shortterm group. The NE tube feeding indications were not statistically different between the groups. Additional unexpected 


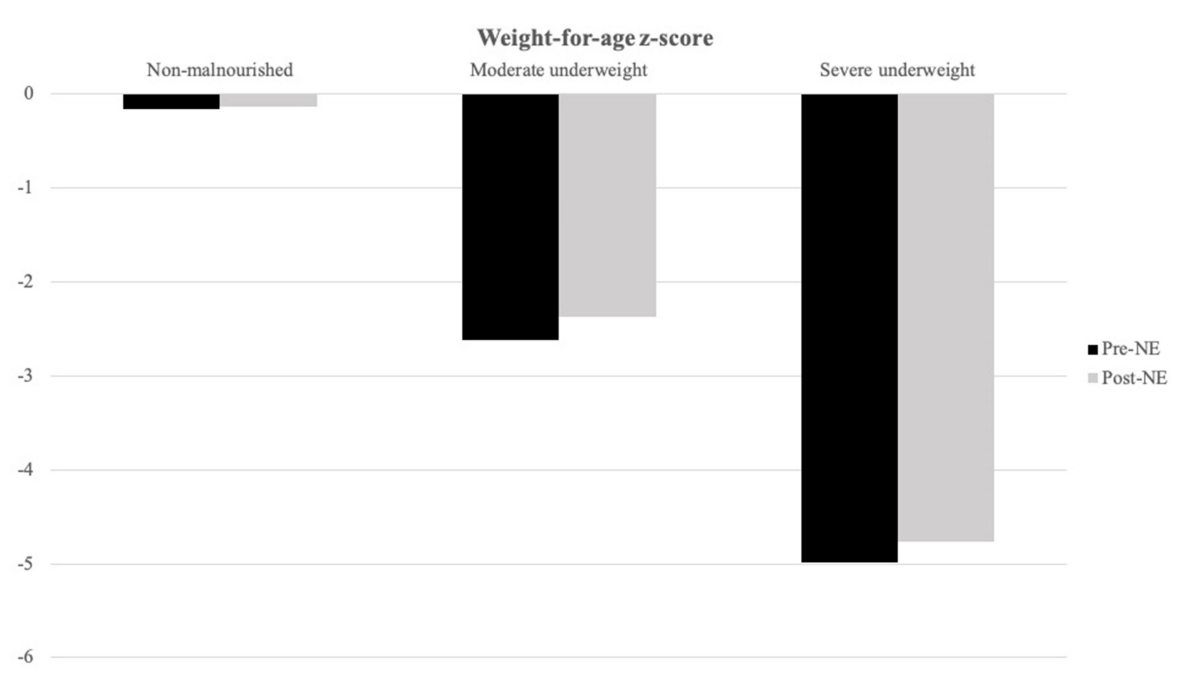

FIGURE 2 | Weight-for-age $z$-score alternations after NE tube placement is stratified by baseline weight-for-age $z$-score. Moderate underweight (baseline weight-for-age $z$-score between -3 and $-2, N=8$ ) and severe underweight group (baseline weight-for-age $z$-score below $-3, N=35$ ) showed better weight-for-age $z$-score increment after NE tube placement than non-malnourished (baseline weight-for-age $z$-score above $-2, N=30$ ) group patients.
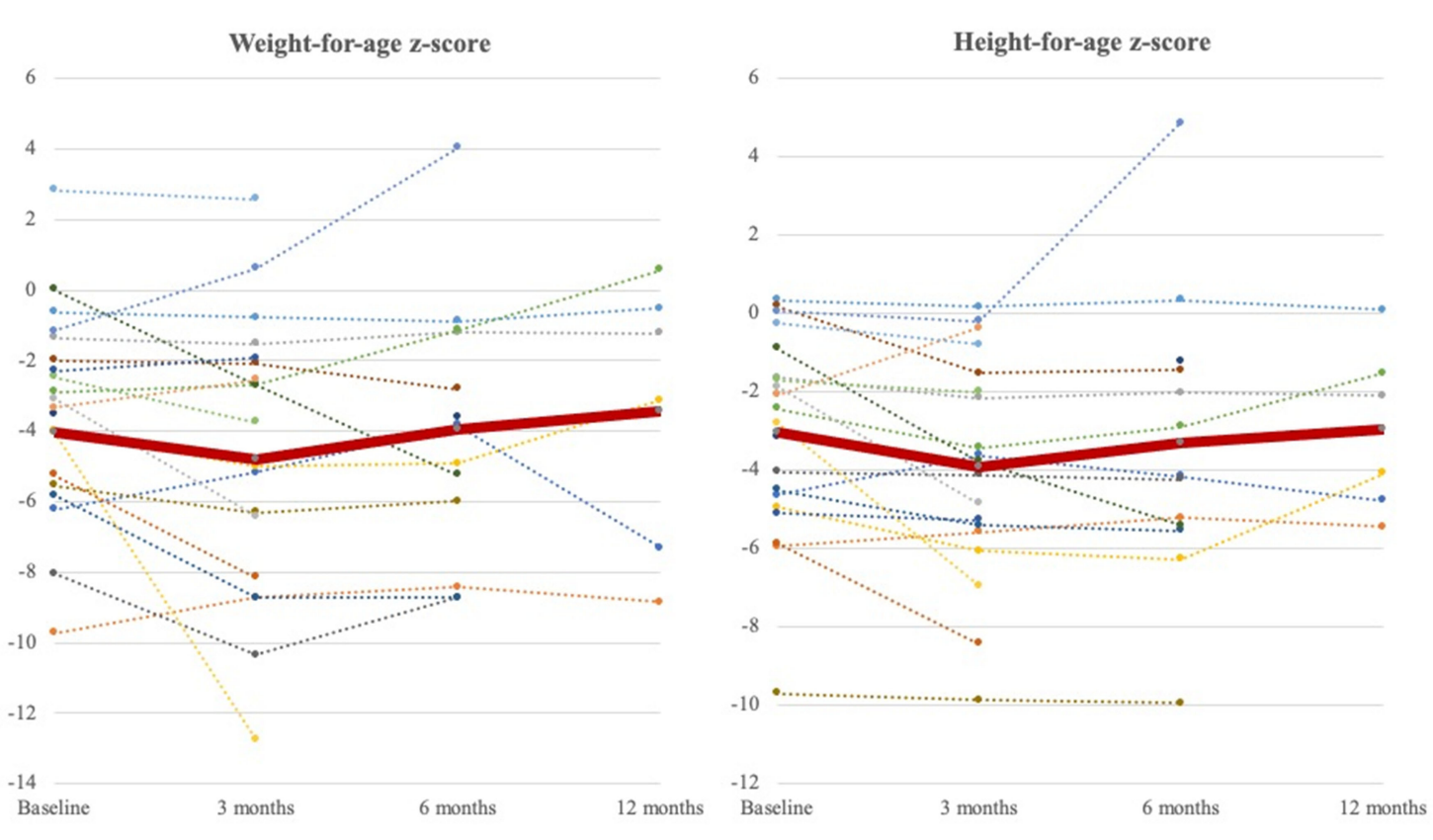

FIGURE 3 | Weight-for-age $z$-score and height-for-age $z$-score curve at 3 months, 6 months, and 1 year during follow-ups. The dot lines represent weight-for-age $z$-score and height-for-age $z$-score of individuals (baseline: $N=20,3$ months; $N=20,6$ months; $N=13,1$ year: $N=6$ ). The thick red line represents the average of all patients. The nadir occurred at 3 months after NE tube placement and increased to exceed baseline until a 1-year follow-up.

$\mathrm{NE}$ tube replacements were required in a long-term group $(1.31 \pm 1.93$ vs. $0.17 \pm 0.43, p<0.001)$. Subsequent requirement of GT or jejunostomy was significantly higher in the long-term group than in the short-term group (24.1 vs. $4.2 \%, p=0.008)$. The long-term group showed a higher increment of weight-for-age $z$-score after the NE tube feeding support.

\section{Nasoenteric Tube-Related Adverse Events}

A total of 47 unexpected reinsertions (26.7\%) were performed due to tube dislodgement $(N=37,78.7 \%)$, tube dysfunction $(N=9,19.1 \%)$, and tube breaks $(N=1,0.02 \%)$. No tube-related bacterial enterocolitis, intussusception, and refeeding syndrome were observed. One major NE tube-related complication was observed in a 2-month-old girl with congenital pulmonary 
TABLE 2 | Clinical outcomes of NE-tube placement.

\begin{tabular}{|c|c|c|c|c|c|}
\hline & All & Severe GERD & Delayed gastric empty & UGI partial obstruction & Post-OP \\
\hline Number & 77 & 42 & 23 & 7 & 5 \\
\hline NE duration (days)* & $133.7 \pm 232.9$ & $161.9 \pm 342.7$ & $117.3 \pm 170.1$ & $96.6 \pm 128.4$ & $27.2 \pm 4.0$ \\
\hline \multicolumn{6}{|c|}{ Subsequent feeding strategy } \\
\hline Oral feeding & $16(20.8 \%)$ & $6(14.3 \%)$ & $2(8.7 \%)$ & $4(57.1 \%)$ & $4(80.0 \%)$ \\
\hline$N G$ & 30 (39.0\%) & $15(35.7 \%)$ & 13 (56.5\%) & $1(14.3 \%)$ & $1(20.0 \%)$ \\
\hline Keep NE & $6(7.8 \%)$ & $3(7.1 \%)$ & $3(13.0 \%)$ & 0 & 0 \\
\hline Gastrostomy/Jejunostomy & $9(11.7 \%)$ & $8(19.0 \%)$ & 0 & $1(14.3 \%)$ & 0 \\
\hline Mortality & $7(9.1 \%)$ & $2(4.8 \%)$ & $4(17.4 \%)$ & $1(14.3 \%)$ & 0 \\
\hline Loss follow-up & $9(11.7 \%)$ & 8 (19.0\%) & $1(4.3 \%)$ & 0 & 0 \\
\hline
\end{tabular}

GERD, gastrointestinal reflux disease; UGl, upper gastrointestinal; OP, operation.

${ }^{*}$ Data present with mean $\pm S D$.

lymphangiectasia. Bowel perforation was found at $4 \mathrm{~cm}$ distal to the ligament of Treitz after NE tube placement for 6 days. The patient expired 32 days later because of ventilator-associated pneumonia. No direct NE tube-related mortality was observed.

\section{DISCUSSION}

This research showed positive clinical outcomes from different aspects for pediatric patients requiring enteral nutrition therapy. Endoscopic-guided NE tube placement is relatively safe, and a high success rate procedure, for pediatric patients.

Children who require tube feeding mostly have multiple medical diagnoses, of which congenital abnormalities (42\%), perinatal problems (38\%), and neurologic diseases (16\%) are the most common comorbidities, as indicated in the Netherlands study (14). Another study in Poland reported neurological disorders (64.2\%) as the most common underlying diseases indicated for tube feeding (15). Neurologic dysfunction, prematurity, and congenital anomalies accounted for 63.6, 32.5, and $18.2 \%$, respectively, of comorbidities in patients of this study. There were 12 cases $(15.6 \%)$ of underlying diseases of prematurity and neurologic dysfunction, and, in this case, tube feeding can improve the nutritional status, drooling, secretion management, and constipation and ease caregiver medication administration and feeding (16).

The procedure of NE tube insertion in this study was safe without complications except for placement failure twice. The success rate of using endoscopic-guided enteric tube was $98.9 \%$ in our institution, similar to that in the previous reports $(5,17)$. The most common complication was tube dislodgement for 37 times, which occurred in 19 patients (24.7\%). The incidence of accidental feeding tube dislodgement $\sim 28.9-40 \%$ in the previous reports $(18,19)$. Tube occlusion episodes totaled nine times in eight patients. Inadequate flushing and administration of more than three kinds of medications may contribute to tube occlusion. Enteral devices into the small bowel had been reported with serious adverse events, including bowel perforation, volvulus, major bleeds, and intussusceptions $(20,21)$. None of the patients exhibited serious adverse events, except for one (1.3\%) who had developed bowel perforation at 6 days post-insertion. No tube-related mortality was observed.

In the present study, the most common indication for NE tube placement is severe GERD (54.5\%), and, overall, $71.4 \%$ of the patients who exhibited improved GERD-symptoms (vomiting, desaturations/bradycardia during/after feeding) after NE tube feeding. Small bowel feeding reduces the risk of aspiration pneumonia in mechanically-ventilated adult patients $(22,23)$ although evidence in pediatric patients is equivocal $(24,25)$. NE tube feeding can reduce aspiration pneumonia episodes in patients of this study.

Delayed gastric emptying, a secondary underlying disease, or sedatives or muscle relaxants can increase the risk of aspiration pneumonia and interrupt enteral feeding (26). More than $50 \%$ of critically ill children can present a high gastric residual volume (26). The European Society for Pediatric Gastroenterology, Hepatology, and Nutrition (ESPGHAN) of the expert group recommends that transpyloric tube feeding may be considered to provide enteral nutrition when gastric feeding fails in critically ill children (6). Nguyen et al. suggested that the first step for treating feeding intolerance in critically ill patients should be the use of prokinetics, such as erythromycin and metoclopramide. If the prokinetic therapy is unsatisfactory, post-pyloric feeding should be considered (27). The use of prokinetics, including metoclopramide and mosapride, had been attempted on patients but with an insufficient response. Thus, post-pyloric tube feeding was initiated. As a result, the average daily feeding volume significantly increased from $144.8 \pm 136.5$ to $1,103.1 \pm 524.7 \mathrm{ml}$ in the delayed gastric emptying group. The feeding volume increment could help to wean from PN support, reduce further septic complications, and reduce the hospitalization days.

Four out of five post-surgery patients were from the burn injury population, and the remaining one had necrotizing fasciitis. Pediatric patients with burns required high levels of nutrition due to body growth and development, high levels of oxidative stress, an intense inflammatory response, and prolonged hypercatabolism (28). The small bowel is the first portion of the GI tract that regains the function of absorption and motility within $6-8 \mathrm{~h}$ post-operation. Nutrition support strategies are suggested as early as possible if enteral feeding 
TABLE 3 | Short- ( $<8$ weeks) and long-term ( $>8$ weeks) use of NE tube feeding.

\begin{tabular}{|c|c|c|c|}
\hline & Short-term & Long-term & $P$-value \\
\hline Number & 48 & 29 & \\
\hline Age (years, mean $\pm S D$ ) & $3.72 \pm 4.83$ & $5.27 \pm 6.23$ & 0.958 \\
\hline Baseline weight-for-age $z$-score & $-2.31 \pm 2.41$ & $-3.41 \pm 3.04$ & 0.088 \\
\hline Baseline height-for-age z-score & $-2.13 \pm 2.69$ & $-2.89 \pm 2.69$ & 0.243 \\
\hline \multicolumn{4}{|l|}{ NE tube placement } \\
\hline Unexpected times & $0.17 \pm 0.43$ & $1.31 \pm 1.93$ & * $<0.001$ \\
\hline \multicolumn{4}{|l|}{ Comorbidity } \\
\hline Neurologic & $26(54.1 \%)$ & 23(79.3\%) & ${ }^{*} 0.026$ \\
\hline Preterm & $15(31.2 \%)$ & $10(34.4 \%)$ & 0.769 \\
\hline \multicolumn{4}{|l|}{ NE tube indications } \\
\hline Partial UGI obstruction & $5(10.4 \%)$ & $2(6.9 \%)$ & 0.603 \\
\hline \multicolumn{4}{|l|}{ Growth } \\
\hline Weight-for-age z-score alternations & $0.11 \pm 0.85$ & $0.21 \pm 1.85$ & 0.782 \\
\hline Height-for-age z-score alternations & $-0.12 \pm 0.56$ & $-0.50 \pm 1.88$ & 0.301 \\
\hline \multicolumn{4}{|l|}{ Outcomes } \\
\hline Keep NE tube feeding & $3(6.3 \%)$ & $3(10.3 \%)$ & 0.516 \\
\hline Oral feeding & $15(31.3 \%)$ & $1(3.4 \%)$ & ${ }^{*} 0.004$ \\
\hline NG tube feeding & $19(39.6 \%)$ & $11(37.9 \%)$ & 0.885 \\
\hline Gastrostomy/Jejunostomy & $2(4.2 \%)$ & 7 (24.1\%) & * 0.008 \\
\hline Mortality & $3(6.3 \%)$ & $4(13.8 \%)$ & 0.265 \\
\hline Loss follow-up & $6(12.5 \%)$ & $3(10.3 \%)$ & 0.775 \\
\hline
\end{tabular}

SD, standard deviation; GERD, gastroesophageal reflux disease; UGl, upper gastrointestinal; NE, Nasoenteric tube; NG, Nasogastric tube.

${ }^{*}$ Numerical data were analyzed using the Student's t-test, and categorical data were analyzed using the Chi-squared test. A p < 0.05 was considered to be statistically significant.

shows no contraindication and can be started in a safe manner via the nasoduodenal tube or the nasojejunal tube until sufficient orally ingested calories are obtained (29). Patients received NE tube insertion within $24-48 \mathrm{~h}$ after surgery, with an insertion duration average of $27.2 \pm 4.0$ days. The albumin level significantly increased from $2.68 \pm 0.67$ to $3.66 \pm 0.33 \mathrm{~g} / \mathrm{dl}$ during this period. A meta-analysis and systemic review study reported that early enteral nutrition provided within $24 \mathrm{~h}$ after injury showed benefits of a reduced duration of hospital stay and low caloric deficit and weight loss but a high incidence of diarrhea and vomiting in pediatric burn patients (30). Hypoalbuminemia in burn patients is strongly associated with burn severity and high mortality rate $(31,32)$.

Our study showed positive nutritional outcomes, including a statistically significant increase in the daily feeding amount and serum albumin level, after NE tube feeding for an average of $133.8 \pm 272.9$ days. Patients with weight-for-age $z$-score and height-for-age $z$-score $<-3$ accounted for 45.5 and $26.0 \%$, respectively, indicating that patients were severely malnourished at the baseline. We further stratified patients into three different baseline weight-for-age $z$-score for evaluation and found that NE tube feeding may help in better improving the weightfor-age $z$-score in the baseline malnourished patients. An appropriate linear growth was not achieved in our study. Compared with other GT (33), percutaneous endoscopic GT (PEG) (34), and the surgical jejunostomy (SJ) studies (35), our patients had similar weight-for-age $z$-score improvement from -4.02 to -3.42 in 1 year in NE tube feeding, from -2.8 to -1.8 in 1 year in GT, from -1.5 to 0.9 in 6 months in PEG, and from -3.7 to -2.6 in 1 year in SJ. In addition, we analyzed the growth of patients who subsequently received surgical GT $(N=8)$ and fundoplication $(N=7)$ and showed a decrease in weight-for-age $z$-score from -2.76 \pm 1.73 to $-3.25 \pm 2.91$ and an increase in height-for-age $z$ score from $-3.34 \pm 2.35$ to $-3.19 \pm 2.60$ during follow-up for $1,164.3 \pm 697.7$ days.

The strengths of this study include a relatively large sample size and the standardization of enteral nutrition management and consultation for surgical approaches, which are based on the guidelines for pediatric gastroenterologists and pediatric surgeons. In addition, this study statistically evaluated factors related to favorable or unfavorable nutritional outcomes in NE tube feeding; such an evaluation was never discussed in previous literature studies. This study has several limitations. First, this work is a retrospective review with an inherent difference in the timing of 
initiation of NE tube feeding among patients with different underlying diseases. Second, nutritional parameters (serum $\mathrm{Hb}$ and albumin) were not checked in several patients because they achieved clinical success. Finally, we did not compare the differences in the nutritional outcomes between patients with long-term NE tube feeding and those with enterostomy.

\section{CONCLUSIONS}

Endoscopic-guided placement of NE tube is a relatively safe, non-invasive procedure for pediatric patients who require enteral nutrition therapy but are intolerable to gastric feeding. The NE tube feeding intervention can significantly increase feeding volumes, reduce clinical symptoms, wean from PN support, and improve growth conditions, even in the baseline severely malnourished patients. NE tube feeding may keep the weight gain steady without any significant adverse event in a longterm use.

\section{DATA AVAILABILITY STATEMENT}

The raw data supporting the conclusions of this article will be made available by the authors, without undue reservation.

\section{REFERENCES}

1. Braegger C, Decsi T, Dias JA, Hartman C, Kolacek S, Koletzko B, et al. Practical approach to paediatric enteral nutrition: a comment by the ESPGHAN committee on nutrition. J Pediatr Gastroenterol Nutr. (2010) 51:110-22. doi: 10.1097/MPG.0b013e3181d336d2

2. Sigalet DL, Mackenzie SL, Hameed SM. Enteral nutrition and mucosal immunity: implications for feeding strategies in surgery and trauma. Can J Surg. (2004) 47:109-16.

3. Volpe A, Malakounides G. Feeding tubes in children. Curr Opin Pediatr. (2018) 30:665-70. doi: 10.1097/MOP.0000000000000666

4. DeLegge MH. Enteral access and associated complications. Gastroenterol Clin North Am. (2018) 47:23-37. doi: 10.1016/j.gtc.2017.09.003

5. Damore LJ II, Andrus CH, Herrmann VM, Wade TP, Kaminski DL, Kaiser GC. Prospective evaluation of a new through-the-scope nasoduodenal enteral feeding tube. Surg Endosc. (1997) 11:460-3. doi: 10.1007/s004649900390

6. Broekaert IJ, Falconer J, Bronsky J, Gottrand F, Dall'Oglio L, Goto E, et al. The use of jejunal tube feeding in children: a position paper by the gastroenterology and nutrition committees of the european society for paediatric gastroenterology, hepatology, and nutrition 2019. J Pediatr Gastroenterol Nutr. (2019) 69:239-58. doi: 10.1097/MPG.0000000000002379

7. Romano C, van Wynckel M, Hulst J, Broekaert I, Bronsky J, Dall'Oglio L, et al. European society for paediatric gastroenterology, hepatology and nutrition guidelines for the evaluation and treatment of gastrointestinal and nutritional complications in children with neurological impairment. J Pediatr Gastroenterol Nutr. (2017) 65:242-64. doi: 10.1097/MPG.0000000000001646

8. Tume LN, Valla FV. A review of feeding intolerance in critically ill children. Eur J Pediatr. (2018) 177:1675-83. doi: 10.1007/s00431-018-3229-4

9. Pearce CB, Duncan HD. Enteral feeding. Nasogastric, nasojejunal, percutaneous endoscopic gastrostomy, or jejunostomy: its indications and limitations. Postgrad Med J. (2002) 78:198-204. doi: 10.1136/pmj.78.918.198

10. Vanek VW. Ins and outs of enteral access. Part 1: short-term enteral access. Nutr Clin Pract. (2002) 17:275-83. doi: 10.1177/0115426502017005275

11. Pedrón Giner C, Martínez-Costa C, Navas-López VM, GómezLópez L, Redecillas-Ferrero S, Moreno-Villares JM, et al. Consensus on paediatric enteral nutrition access: a document approved by

\section{ETHICS STATEMENT}

The studies involving human participants were reviewed and approved by Ethics Committee of Chang Gung Memorial Hospital (Ref. 202001377B0). Written informed consent from the participants' legal guardian/next of kin was not required to participate in this study in accordance with the national legislation and the institutional requirements.

\section{AUTHOR CONTRIBUTIONS}

C-CC, H-CC, M-WL, and P-JY: diagnosis and management of the patients. M-CC and H-CC: writing of the manuscript, data analysis and interpretation, and final approval of the manuscript. H-CC: critical evaluation and revision of the manuscript. All authors contributed to the article and approved the submitted version.

\section{ACKNOWLEDGMENTS}

We thank all of the children and their parents or caregivers for participating in this study, and we also thank the medical staff (medical residents, medical interns, and nurses) for their clinical care.

SENPE/SEGHNP/ANECIPN/SECP. Nutr Hosp. (2011) 26:1-15. doi: 10.3305/nh.2011.26.1.4647

12. Bharadwaj S, Ginoya S, Tandon P, Gohel TD, Guirguis J, Vallabh H, et al. Malnutrition: laboratory markers vs nutritional assessment. Gastroenterol Rep (Oxf). (2016) 4:272-80. doi: 10.1093/gastro/ gow013

13. Lord LM. Enteral access devices: types, function, care, and challenges. Nutr Clin Pract. (2018) 33:16-38. doi: 10.1002/ncp.10019

14. Krom H, van Zundert SMC, Otten MGM, van der Sluijs Veer L, Benninga MA, Kindermann A. Prevalence and side effects of pediatric home tube feeding. Clin Nutr. (2019) 38:234-9. doi: 10.1016/j.clnu.2018.01.027

15. Szlagatys-Sidorkiewicz A, Popińska K, Toporowska-Kowalska E, Borkowska A, Sibilska M, Gebora-Kowalska B, et al. Home enteral nutrition in children-2010 nationwide survey of the polish society for clinical nutrition of children. Eur J Pediatr. (2012) 171:719-23. doi: 10.1007/s00431-0111646-8

16. Mahant S, Friedman JN, Connolly B, Goia C, Macarthur C. Tube feeding and quality of life in children with severe neurological impairment. Arch Dis Child. (2009) 94:668-73. doi: 10.1136/adc.2008.149542

17. Kwauk ST, Miles D, Pinilla J, Arnold C. A simple method for endoscopic placement of a nasoduodenal feeding tube. Surg Endosc. (1996) 10:6803. doi: 10.1007/BF00188530

18. Meer JA. Inadvertent dislodgement of nasoenteral feeding tubes: incidence and prevention. JPEN J Parenter Enteral Nutr. (1987) 11:187-9. doi: 10.1177/014860718701100219

19. Mion LC, Minnick AF, Leipzig R, Catrambone CD, Johnson ME. Patient-initiated device removal in intensive care units: a national prevalence study. Crit Care Med. (2007) 35:2714-20; quiz 25. doi: 10.1097/01.CCM.0000291651.12767.52

20. Maple JT, Petersen BT, Baron TH, Gostout CJ, Wong Kee Song LM, Buttar NS. Direct percutaneous endoscopic jejunostomy: outcomes in 307 consecutive attempts. Am J Gastroenterol. (2005) 100:26818. doi: 10.1111/j.1572-0241.2005.00334.x

21. Al-Zubeidi D, Bishop WP, Rahhal RM. Identifying small bowel intussusception related to a gastroenteric feeding tube. Frontline Gastroenterol. (2011) 2:63-4. doi: 10.1136/fg.2009.001305 
22. Metheny NA, Stewart BJ, McClave SA. Relationship between feeding tube site and respiratory outcomes. JPEN J Parenter Enteral Nutr. (2011) 35:34655. doi: 10.1177/0148607110377096

23. Zhang Z, Xu X, Ding J, Ni H. Comparison of postpyloric tube feeding and gastric tube feeding in intensive care unit patients: a meta-analysis. Nutr Clin Pract. (2013) 28:371-80. doi: 10.1177/0884533613485987

24. Sönmez Düzkaya D, Yildiz S. Effect of two different feeding methods on preventing ventilator associated pneumonia in the paediatric intensive care unit (PICU): a randomised controlled study. Aust Crit Care. (2016) 29:13945. doi: 10.1016/j.aucc.2015.11.001

25. McClave SA, Martindale RG, Rice TW, Heyland DK. Feeding the critically ill patient. Crit Care Med. (2014) 42:260010. doi: 10.1097/CCM.0000000000000654

26. Martinez EE, Pereira LM, Gura K, Stenquist N, Ariagno K, Nurko S, et al. Gastric emptying in critically Ill children. JPEN J Parenter Enteral Nutr. (2017) 41:1100-9. doi: 10.1177/0148607116686330

27. Nguyen NQ. Pharmacological therapy of feed intolerance in the critically ills. World J Gastrointest Pharmacol Ther. (2014) 5:148-55. doi: 10.4292/wjgpt.v5.i3.148

28. Trocki O, Michelini JA, Robbins ST, Eichelberger MR. Evaluation of early enteral feeding in children less than 3 years old with smaller burns (8-25 per cent TBSA). Burns. (1995) 21:17-23. doi: 10.1016/0305-4179(95)90775-U

29. Lee JO, Benjamin D, Herndon DN. Nutrition support strategies for severely burned patients. Nutr Clin Pract. (2005) 20:32530. doi: 10.1177/0115426505020003325

30. Valentini M, Seganfredo FB, Fernandes SA. Pediatric enteral nutrition therapy for burn victims: when should it be initiated? Rev Bras Ter Intensiva. (2019) 31:393-402. doi: 10.5935/0103-507X.20190062

31. Aguayo-Becerra OA, Torres-Garibay C, Macías-Amezcua MD, FuentesOrozco C, Chávez-Tostado Mde G, Andalón-Dueñas E, et al. Serum albumin level as a risk factor for mortality in burn patients. Clinics (Saõ Paulo). (2013) 68:940-5. doi: 10.6061/clinics/2013(07)09

32. Pérez-Guisado J, de Haro-Padilla JM, Rioja LF, Derosier LC, de la Torre JI. Serum albumin levels in burn people are associated to the total body surface burned and the length of hospital stay but not to the initiation of the oral/enteral nutrition. Int J Burns Trauma. (2013) 3:15963. doi: $10.1155 / 2013 / 707360$

33. Fortunato JE, Troy AL, Cuffari C, Davis JE, Loza MJ, Oliva-Hemker $\mathrm{M}$, et al. Outcome after percutaneous endoscopic gastrostomy in children and young adults. J Pediatr Gastroenterol Nutr. (2010) 50:390-3. doi: 10.1097/MPG.0b013e3181aed6f1

34. $\mathrm{Wu} \mathrm{FY}$, Wu JF, Ni YH. Long-term outcome after percutaneous endoscopic gastrostomy in children. Pediatr Neonatol. (2013) 54:326-9. doi: 10.1016/j.pedneo.2013.04.008

35. Singh RR, Eaton S, Roebuck DJ, Barnacle AM, Chippington S, Cross KMK, et al. Surgical jejunostomy and radiological gastro-jejunostomy tube feeding in children: risks, benefits and nutritional outcomes. Pediatr Surg Int. (2018) 34:951-6. doi: 10.1007/s00383-018-4303-8

Conflict of Interest: The authors declare that the research was conducted in the absence of any commercial or financial relationships that could be construed as a potential conflict of interest.

Copyright (c) 2021 Chen, Chao, Yeh, Lai and Chen. This is an open-access article distributed under the terms of the Creative Commons Attribution License (CC BY). The use, distribution or reproduction in other forums is permitted, provided the original author(s) and the copyright owner(s) are credited and that the original publication in this journal is cited, in accordance with accepted academic practice. No use, distribution or reproduction is permitted which does not comply with these terms. 